\title{
Editorial
}

\section{POST-SECONDARY EDUCATION IN NORTHERN CANADA}

\section{PETER FERRIS*}

The problems that face educators who are involved with the provision of post-secondary education in northern Canada are generally well known. There is the challenge of geography; of providing services across huge land masses to a relatively small and dispersed, and often isolated, population. That population, although relatively small in number, requires a range of educational services, from literacy to professional training, which adds special challenges to the question of how educational provision may be most effectively organized. Also, it may be argued that educational systems in the past have failed, in terms of cultural appropriateness and sensitivity, to meet the needs of aboriginal people who, as a result, often lack standard prerequisites for entry into post-secondary training and education programmes. Further, education in the north has to be concerned with more than individual attainment. There is a perception on the part of aboriginal people that education is an indespensable tool for help fashion community development, indigenous control over a range of services, and self-government. Not surprisingly these ambitions are accompanied by a desire to shape the form of education provision and to influence the manner of its delivery. Finally, the desire of northerners, both aboriginal and non-aboriginal, to have greater access to employment opportunities associated with resource extraction and development activities requires special commitments, educational and political, if they are to be realized.

With a few notable exceptions it has taken years to recognize that moving to the south in order to take advantage of post-secondary opportunities is not an option for most northerners. Equally, providing programmes and applying policies in the north that are based on southern imperatives creates dissonance and failure. Northern education is a field wherein even the most blinkered administrator must see that normal administrative devices will not work, where the most hide-bound decision maker must be sensitive to local political sensibilities, and the most miserly holder of the purse strings must admit that a reasonable return on the dollar of educational investment can only be obtained by having the courage of what is, in the final analysis, an educational conviction. Those who do not grasp these realities conceptually soon have the point made for them experientially. Isolated communities with poor housing, minimal physical infrastructure, and very high levels of unemployment do not present a fruitful context for educational 
endeavour; racism, whether in the form of outright bigotry, or in a more subtle manifestation, still affects decision making; communities based on boom or bust resource economies lack stability; and many jobs that potentially could be filled by northerners continue to fall to a mobile southern based labour force.

Thus, the north is very much a special case. However, it would be wrong to depict an educational wasteland unrelieved by a prospect of progress and development. In fact, if one can reach beyond the problems, without ever underestimating them, it becomes clear that there is much to appreciate in northern education. There is considerable evidence of innovative, and sound, educational policy. Too often this goes unremarked. Thus, the purpose of this editorial is to extend the awareness of educators of policies and programmes which have been successful and effective. There is another reason, even more compelling to the author, and that is to celebrate the achievements of aboriginal northerners many of whom have attained high levels of educational accomplishment in the face of often overwhelming odds.

In that region with which the writer is most familiar, that is northern Manitoba, a quiet revolution has occurred over the last twenty years. Over 400 fully qualified native teachers have graduated, as well as nurses, social workers, lawyers, pharmacists, doctors, and B.A. and B.Sc. graduates. At the para-professional level training has been provided and employment attained, in areas such as health care, child care, probation and correctional services, and alcohol and drug counselling. A major initiative to provide education and training programmes allied to the construction of the Limestone generating station, designed to maximize the employment of northerners in general and aboriginal northerners in particular on the project, has produced an aboriginal participation rate that has ranged between $18 \%$ and $33 \%$ of the total workforce, and, equally important, qualified, skilled, productive tradespeople. This project will also see, within the next two years, aboriginal graduates in civil and electrical/electronic technology and degree engineers. It may be useful to isolate the key elements of these initiatives.

The successful graduates in the areas listed above lacked all the normal pre-conditions of success. Very few are high school graduates, and those that were lacked university entrance standing. All were native students, many with dependents, often single parents, without financial resources. Many, but by no means all, were required to leave their home community to pursue their academic ambitions. This set of circumstances describes the reality of many northerners, and too often is the determinant of automatic failure rather than success. Thus, any response has to be comprehensive, and has to provide adequate financial support and personal assistance as well as appropriate academic services. Providing academic upgrading within the context of a programme rather than as yet another prerequisite has been found to be extremely successful. For example, it becomes immediately apparent to a student who is studying engineering why it is necessary to understand and apply a particular mathematical concept. Thus academic 
upgrading becomes a means to an end, in the short term in solving a problem and in the foreseeable future in obtaining a degree, rather than a somewhat mysterious end in itself. Appropriate academic upgrading is made available by stretching the course of studies, extending the academic year to 11 months, and often extending the degree course by one year. The commitment required by the student is considerable, but there is no lack of candidates, and success rates, as measured by the number of students graduating as a percentage of those enrolling is equal to that of "regular" students.

It is essential to note that these initiatives have been successful not only in terms of producing graduates who can make an important contribution to their community, but also because usual standards of attainment are not compromised and the graduate teacher, social worker, carpenter possess a degree, diploma, or journeyman's certificate which is not equivalent to but exactly the same as that held by a southern graduate. The achievement of recognized standards of attainment by individuals who very often lack the usual prerequisites for entry is a result of the application of sound educational policy. Educators have known for sometime that high school marks are often unreliable predicators of subsequent attainment. In their absence educators must use other criteria, admittedly difficult to quantify, such as maturity, commitment to self development, and need. Integrating academic upgrading into an extended academic program while ensuring that the upgrading is appropriate to course work has proved to be a successful means of overcoming knowledge gaps. This approach has also to be encompassed within a program design that not only provides extra academic supports, such as tutoring, but personal supports for the student and often the student's family, a package that must include adequate financial assistance.

The commitment to mount these kinds of "special" programs, which may have a premium of $25 \%$ of normal costs, is based on an expectation that students will succeed and thus justify the extra costs involved. There is ample evidence to indicate this is the case. In fact, the financial benefits of such human development investments are significant: individuals, and families, moved from being recipients of welfare to permanent taxpayers; salary dollars are more likely to remain and circulate in and through the local and regional economies than drain south. Also, of course, as our colleagues in education psychology have been telling us for years, any new student who is greeted with the expectation that he or she will succeed is likely to do so. This is an expectation that most northern students have never experienced.

The involvement of northern communities in the determination of their own futures is a political reality that cannt, and should not, be ignored. Post-secondary education and training is vital to the development of indigenous human service, technical, and physical infrastructures. In many cases these infrastructures are only now being developed; in other cases, such as local control of public school education and local control of child welfare services, alien systems, remotely controlled from urban centres by bureaucracies insensitive to local conditions, are 
being dismantled and replaced. It is not sufficient, and it is ineffective, for educational institutions to offer programming that is based on institutional imperatives and curricula relevant for urban centres.

The involvement of northern representatives as partners in the determination of policy and in the design of programs and delivery system is vital if educational provision is to be deemed appropriate and relevant to its consumers. Even where southern institutions demonstrate a commitment to the provision of educational opportunity for northerners it is understandable that such a commitment will never be seen as central to their prime mission. Political commitment is often needed to establish separate funding arrangements for northern programming to ensure adequate financial support. The creation of new institutions in the North undoubtedly helps to address the issue of geographic access; however, if such institutions are bureaucratically linked to southern bases then the advantage is qualified.

The fulcrum around which northern educational provision should be determined is the northern community. Typically, this is relatively isolated and relatively small in size. Yet the range of need, in terms of education alone, is enormous, and ranges from basic literacy to degree level programming, and, in between, contains particular and specific requirements, for example, a technician to maintain sewer and water services, a journeyperson carpenter to oversee house construction. The needs of northern communities based on resource extraction differ only in degree.

Given the necessary commitment, both political and financial, and new organizational structures, these needs can be addressed. There are successful models of regionally delivered professional training programs. Of even greater significance there are successful models of community based professional training programs as well as trades training initiatives that require a minimal amount of time spent outside the community.

In summary, northern education is an area of immense challenge; an area where the need for relevant education as a means of individual, community, and regional realization of potential is so patent that anything less than a full commitment to it is not so much a cost saving in a ledger book as perpetuation of indignity; an area where it would still be possible to fill a journal with a litany of failed policy, inadequate commitment, and short-sighted programming which merely adds to the weight of hopelessness, despair, and social anomie. There is only one reason for taking a different course and that is to demonstrate and celebrate the immense achievements of those northerners, particularly aboriginal northerners, who have succeeded in scaling the walls of academic attainment. That those walls, to some educators, are built on questionable foundations and are of suspect design does not diminish their power to exclude. As educators we should be inspired by the energy, ambition, and ability that it takes to climb them, and, at the very least, we should be prepared to advocate and design scaling ladders which will bear the weight of considerably greater numbers of students. 


\section{L'ENSEIGNEMENT POST-SECONDAIRE DANS LE NORD CANADIEN}

\section{PETER FERRIS}

Les problèmes qui se posent aux éducateurs qui dispensent l'enseignement post-secondaire dans le Nord canadien sont généralement bien connus. Il y a le défi géographique d'offrir des services sur une superficie immense, à une population relativement petite et dispersée, et souvent isolée. Bien que cette population soit relativement petite, elle exige toute une gamme de services d'enseignement allant de l'alphabétisation à la formation professionnelle, ce qui ajoute au problème de trouver quelle peut être la manière la plus efficace d'organiser la prestation des services d'enseignement. On peut également soutenir que sur les plans de la convenance et de la sensibilisation à la culture, les systèmes d'éducation établis dans le passé n'ont pas réussi à répondre aux besoins des aborigènes qui, par conséquent, souvent ne remplissent pas les conditions d'admission aux programmes de formation et d'enseignement post-secondaires. De plus, dans le Nord, l'éducation ne doit pas se limiter à l'enrichissement personnel. Les aborigènes perçoivent l'éducation comme un outil indispensable pour façonner leur développement communautaire, contrôler toute une gamme de services et asseoir leur gouvernement autonome. Il n'est pas surprenant que ces ambitions s'accompagnent d'un désir de façonner les services d'enseignement et d'influer sur le mode de prestation de ces services. Finalement, la volonté des Nordistes tant aborigènes que non aborigènes d'avoir un plus grand accès aux emplois liés à l'extraction de richesses naturelles et aux activités de développement exige des engagements spéciaux de la part du monde de l'éducation et des politiciens pour devenir réalité.

A part quelques exceptions remarquables, il a fallu des années pour qu'on reconnaisse que le fait de déménager au sud pour profiter de possibilités d'enseignement post-secondaire ne constitue pas une solution pour la plupart des gens du Nord. De même, la mise en oeuvre dans le Nord de programmes et de politiques fondés sur les exigences du sud se traduit par la mésentente et l'échec. Dans le Nord, l'éducation constitue un domaine où même l'administrateur aux vues les plus étroites doit voir que les mécanismes administratifs courants ne fonctionnent pas, où le décideur le plus borné doit être ouvert aux susceptibilités politiques locales, et où le teneur des cordons de la bourse le plus avare doit admettre qu'on ne peut pas obtenir un rendement raisonable des dollars investis dans l'éducation à moins d'avoir le courage de ce qui est, en fin d'analyse, une conviction éducationnelle. Ceux qui ne saisissent pas le concept de ces réalités l'apprennent rapidement par l'expérience. Des collectivités isolées aux logements inadéquats, à l'infrastructure physique minimale et au taux de chômage très élevé ne présentent pas un contexte propice à l'éducation; le racisme, qu'il se manifeste sous forme de sectarisme pur ou sous une forme plus subtile, influe sur la prise de décisions; les collectivités dont l'économie dépend des périodes d'expansion ou de 
récession des richesses naturelles manquent de stabilité; et la main-d'oeuvre mobile provenant du sud continue à s'accaparer une foule d'emplois que pourraient occuper les gens du Nord.

Ainsi, le Nord présente un cas très particulier. Il serait toutefois injuste de dépeindre un désert éducationnel sans perspective de progrès et d'évolution. Lorsqu'on réussit à s'élever au-dessus des problèmes, sans jamais les sousestimer, il devient clair que l'éducation dans le Nord offre beaucoup à apprécier. De fait, il existe de nombreux signes d'une politique à la fois innovatrice et sensée en matière d'enseignement, ce qui, trop souvent, passe inaperçu. Le but de cet éditorial est donc de sensibiliser davantage les éducateurs aux politiques et aux programmes qui ont connu du succès tout en s'avérant efficaces. Il y a aussi une autre raison encore plus importante pour l'auteur qui veut acclamer les succès des aborigènes du Nord, dont beaucoup ont atteint un niveau d'instruction élevé malgré des obstacles souvent écrasants.

Au cours des vingt dernières années, une révolution tranquille s'est produite dans cette région que l'auteur connaît très bien, le nord du Manitoba. Ici, plus de 400 enseignants autochtones qualifiés ont obtenu leur diplôme, de même des infirmières, des travailleurs sociaux, des avocats, des pharmaciens, des médecins et des détenteurs d'un B.A. ou d'un B. Sc. Sur le plan para-professionnel, on a fourni de la formation qui a mené à l'obtention d'un emploi dans des domaines tels que les soins de santé, la protection de l'enfance, la probation et les services correctionnels ainsi que les services d'aide aux alcooliques et aux toxicomanes. Un important projet visant à fournir des programmes d'enseignement et de formation s'ajoutant à la construction de la centrale électrique Limestone, et conçu de manière à maximiser l'emploi des gens du Nord en général et des aborigènes du Nord en particulier sur le chantier, a engendré un taux de participation des aborigènes variant de $18 \%$ à $33 \%$ de l'ensemble de la main-d'oeuvre. Tout aussi important est le fait que ce projet a également produit des gens de métier qualifiés, compétents et productifs. Toujours grâce à ce projet, des aborigènes obtiendront d'ici deux ans des diplômes d'ingénieurs en génie civil, en électronique et en électrotechnique. Vous trouverez ailleurs dans ce numéro une description détaillée de ce projet ainsi que des exposés sur des programmes qui ont connu le succès dans d'autres régions septentrionales canadiennes. Il peut toutefois être utile de mettre en évidence les éléments clés de ces initiatives.

Les gens ayant obtenu un diplôme dans un domaine cité précédemment ne remplissaient aucune des conditions normalement requises pour réussir. Très peu d'entre eux avaient complété leurs études secondaires, et ceux qui les avaient complétées ne répondaient pas aux conditions d'admission de l'université. Tous étaient des étudiants indigènes sans ressources financières, et beaucoup avaient des personnes à charge et, dans bien des cas, ils étaient des parents célibataires. Beaucoup, mais sûrement pas tous, ont dû quitter leur collectivité d'appartenance afin de poursuivre leur ambition de s'instruire. Cet ensemble de circonstances décrit la réalité de bien des gens du Nord, tout en constituant trop souvent la cause déterminante de l'échec systématique plutôt que du succès. Toute solution doit 
donc être globale et fournir une aide personnelle et un appui financier adéquats ainsi que des services d'enseignement appropriés. On a connu un énorme succès en assurant le perfectionnement scolaire dans le cadre d'un programme plutôt que d'en faire une autre condition préalable. Par exemple, l'étudiant qui étudie le génie voit immédiatement pourquoi il est nécessaire de comprendre et de mettre en application un concept mathématique donné. Ainsi, le perfectionnement scolaire devient pout l'étudiant un moyen d'atteindre un but, soit de résoudre un probème à court terme et d'obtenir un diplôme dans un avenir prévisible plutôt que de constituer une fin en soi quelque peu mystérieuse. Une telle possibilité est offerte aux étudiants en prolongeant l'année scolaire à 11 mois, et, souvent, en prolongeant également le programme d'études d'un an. Bien que cela exige un engagement considérable de la part des étudiants, il ne manque pas de candidats, et le taux de réussite est égal à celui des étudants "réguliers" lorsqu'on l'établit comme pourcentage d'étudiants inscrits à un programme qui obtiennent leur degré.

Il est essentiel de souligner que ces initiatives ont connu du succès non seulement parce qu'il en est sorti des diplômés qui peuvent apporter une importante contribution à leur collectivité, mais aussi parce que le niveau de connaissances acquises normalement exigé est respecté. Ainsi, l'enseignant, le travailleur social ou le charpentier qui termine ses études obtient un grade ou un diplôme universitaire ou, encore, un certificat d'ouvrier spécialisé qui n'est pas équivalent de ce qu'obtient le diplômé du sud, mais qui est bel et bien la même chose. C'est grâce à la mise en pratique de politiques d'enseignement valables que des personnes atteignent un niveau d'études reconnu même si, souvent, elles ne remplissent pas les exigences d'admission habituelles. Les éducateurs savaient depuis quelque temps déjà que les notes obtenues au secondaire ne constituent pas des indices fiables pour prédire les réussites subséquentes. Faute de telles notes, les éducateurs doivent se servir d'autres critères tels la maturité, le besoin et le désir de se perfectionner, critères qui, de l'aveu général, sont difficiles à quantifier. L'expérience nous a montré qu'un moyen efficace de combler les manques de connaissances consiste à intégrer les cours de perfectionnement scolaire à un programme d'études prolongé tout en assurant que le perfectionnement convient à la matière des cours offerts. Cette méthode d'enseignement doit également s'inscrire dans un programme qui prévoit non seulement des ressources d'apprentissage additionnelles, telles des cours de soutien, mais aussi des appuis personnels destinés à l'étudiant et, souvent, à sa famille. Le tout doit constituer un programme global qui comprend également une aide financière adéquate.

L'engagement de mettre en oeuvre ce genre de programmes "spéciaux", qui peuvent coûter jusqu'à $25 \%$ de plus que les programmes ordinaires, se fonde sur l'espoir que les étudiants réussiront, justifiant ainsi le coût supplémentaire. De nombreux indices appuient cette hypothèse. En fait, les avantages financiers de tels investissements dans le perfectionnement humain sont considérables. Des personnes, et des familles, qui dépendaient de l'aide sociale deviennent des contribuables permanents. Aussi, il y a plus de chances que les salaires versés 
restent dans les économies locales et régionales et qu'ils y circulent plutôt que d'être drainés vers le sud. De plus, conformément à ce que nous disent depuis des années nos collègues spécialisés dans la psychopédagogie, tout nouvel étudiant qui est accueilli avec l'espoir qu'il réussisse, espoir que la plupart des étudiants du Nord n'ont jamais connu, réussira probablement.

On ne peut pas et ne doit pas ignorer la réalité politique voulant que les collectivités septentrionales participent à la détermination de leur propre avenir. L'enseignement et la formation post-secondaires sont indispensables à l'élaboration d'infrastructures techniques et physiques pour des services à la personne locaux. Dans bien des cas, on entame à peine,l'élaboration de ces infrastructures. Dans d'autres cas, des systèmes extérieurs dirigés à partir de centres urbains éloignés par des bureaucraties insensibles à la situation locale se font démanteler et remplacer. C'est le cas du contrôle du système d'éducation public et des services de bien-être de l'enfance. Il ne suffit pas et il est inefficace que les établissements d'enseignement offrent des programmes fondés sur des exigences institutionnelles et des programmes d'études qui conviennent aux centres urbains.

Il est indispensable que des représentants du Nord participent à l'établissement des politiques et à la conception des programmes et des systèmes d'application si nous voulons que l'enseignement convienne aux personnes qui en bénéficient tout en répondant à leurs besoins. On peut comprendre que même dans les cas où des établissements du sud font preuve de bonne foi dans l'offre de possibilités d'instruction aux gens du Nord, un tel engagement ne constituera jamais un élément central de leur mission fondamentale. Souvent, afin d'assurer un soutien financier adéquat, un engagement politique est nécessaire afin d'établir des mesures de financement distinctes pour les programmes septentrionaux. La création de nouveaux établissements dans le Nord aide sans doute à alléger le problème de l'accessibilité géographique. Lorsque ces établissements sont bureaucratiquement liés à des bases situées dans le sud cependant, l'avantage s'atténue.

La collectivité septentrionale doit servir de pivot aux services d'enseignement dans cette région. Typiquement, cette collectivité est relativement isolée et petite. Cependant, sur le seul plan de l'éducation l'étendue des besoins est énorme : ils vont de l'alphabétisation élémentaire aux programmes menant à un grade, en passant par des exigences particulières et précises, comme former un technicien qui assurera l'entretien des services d'eau et d'égout, ou un charpentier compagnon qui surveillera la construction d'une maison. Les besoins des collectivités septentrionales dont l'économie repose sur l'extraction des richesses naturelles ne diffèrent qu'en leur degré d'importance.

Avec l'engagement requis sur les plans tant politique que financier, et de nouvelles structures organisationnelles, on peut répondre à ces besoins. Il existe des modèles de programmes de formation professionnelle offerts à l'échelon régional qui ont réussi. Encore plus important, il y a également des modèles de programmes de formation professionnelle axés sur la collectivité qui fonctionnent 
bien, ainsi que des projets de formation technique qui demandent aux participants de ne passer qu'un minimum de temps à l'extérieur de leur collectivité.

En résumé, l'éducation dans le Nord constitue un domaine qui présente un immense défi, un domaine où le besoin d'une instruction pertinente en tant que moyen pour les particuliers, les collectivités et les régions de réaliser leur pleine mesure est tellement évident que n'importe quoi de moins qu'un engagement intégral à son endroit devient beaucoup plus une perpétuation de l'indignité qu'une réduction des coûts dans un grand livre, un domaine où il serait encore possible de remplir un journal d'une litanie de politiques ayant échoué, qu'alourdir le désespoir et l'anomie sociale. Il n'existe qu'une seule raison pour adopter une nouvelle orientation : montrer et acclamer les immenses réalisations des gens du Nord, et plus particulièrement des aborigènes du Nord, qui ont réussi à escalader les murs de la réussite scolaire. Que ces murs soient, selon certains éducateurs, érigés sur des fondations douteuses tout en étant d'une conception suspecte ne réduit en rien leur capacité d'exclusion. En tant qu'éducateurs, nous devrions nous inspirer de l'énergie, de l'ambition et des aptitudes requises pour les escalader, et nous devrions au moins être prêts à concevoir et à recommander des échelles d'escalade qui supporteront le poids d'un nombre beaucoup plus grand d'étudiants. 